\title{
Chromospheres and Winds of Cool Stars
}

Kenneth G. Carpenter

Laboratory for Astronomy and Solar Physics

Code 681, NASA - Goddard Space Flight Center

Greenbelt, MD 20771

\section{SUMMARY}

I present data acquired during the early operations era of the HST with the Goddard High Resolution Spectrograph (GHRS) on three late-type giant and supergiant stars: $\alpha$ Tau (K5 III non-coronal), $\gamma$ Dra (K5 III hybrid), and $\alpha$ Ori (M2 Iab). Figure 1 shows fits to echelle $(R=100,000)$ line profiles of three ions seen in the spectrum of $\alpha$ Tau. The Co II line can be fit with a single gaussian, the self-reversed Fe II line with a combination of an emission and absorption gaussian, but the unreversed C II lines require two emission gaussians of substantially different FWHM and maxima. The complex shape of the C II profiles suggests that the turbulent velocity distribution in the chromosphere is anisotropic (e.g. Gray 1988) or that the lines are formed in two regions characterized by significantly different $\mathrm{T}_{\text {eff }}$ and $\mathrm{v}_{\text {turb }}$, as in the hybrid star models of Harper (1991).

Figure 2 shows low $(R=2000)$, medium $(R=20,000)$, and high $(R=100,000)$ resolution spectra (left) of the O I (UV 2) lines in $\gamma$ Dra and an overplot in velocity space of the echelle O I line profiles (right). In the low resolution data the lines are strongly blended, while at medium resolution the lines are separated but not fully resolved. Finally at high resolution the details in the line profiles are resolved. The second plot illustrates the high density of information which is contained in high signal/noise high-resolution line profiles obtain with the GHRS. The 1302, 1304, and $1306 \AA$ lines are overplotted, but are clearly distinguished on the blue side, where the lines increase in flux as one examines them in wavelength order. Interstellar absorption is seen in the core of the $1302 \AA$ line, but not in the other two lines which have slightly elevated lower energy levels. Absorption by $\mathrm{O} I$ in the stellar wind is seen from zero velocity all the way out to the terminal wind velocity of $70 \mathrm{~km} / \mathrm{s}$ in the $1302 \AA$ line. The wind absorption is also seen in the other two lines, but their weaker intrinsic strength and narrower emission prevents us from seeing all the way out to the terminal velocity. Absorption by $S$ I (UV 9) is seen near the center of the $1306 \AA$ line, confirming the Brown and Jordan (1980) O I - S I fluorescence mechanism. The narrow absorption in the red wing of all three lines is either due to infalling O I near the star or O I in the earth's atmosphere (i.e. telluric O I). 
Figure 3 shows the short-wavelength (far-UV) region of $\alpha$ Ori and $\gamma$ Dra, as observed by IUE and GHRS in their respective low resolution modes. The extreme O I (UV 2) flux deficiency in the $\alpha$ Ori spectrum is clearly seen by comparing the IUE spectra shown in the upper two panels of the first plot (left). GHRS G140L spectra (lower panel of first plot) confirms that the O I, but not the S I, is missing from the supergiant's spectrum. The second plot (right) shows the clear detection of a strong far-UV continuum in place of the expected forest of weak chromospheric emission lines. This continuum is likely formed by recombination in the chromosphere of ions such as Si I, S I, and C I. Superposed on this continuum is a series of very strong, regularly-spaced absorption features due to the $4^{\text {th }}$-positive A-X system of CO located in the well-known circumstellar shell of Betelgeuse. These two detections will provide with us with additional unexpected diagnostics of the chromosphere and circumstellar shell of this star and illustrate well the value of serendipity in science.

Additional discussion of these and other HST observations of cool stars can be found in Carpenter (1991).

\section{REFERENCES}

1 Brown, A. and Jordan, C. 1980, M.N.R.A.S., 191, 37P.

2 Carpenter, K. G. 1991, in Cool Stars, Stellar Systems, and the Sun, in press.

3 Gray, D. F. 1988, chap. 1 in Lectures on spectral Line Analysis: F, G., and K Stars, (The Publisher: Arva, Ontario).

4 Harper, G. 1991, M.N.R.A.S., , submitted.
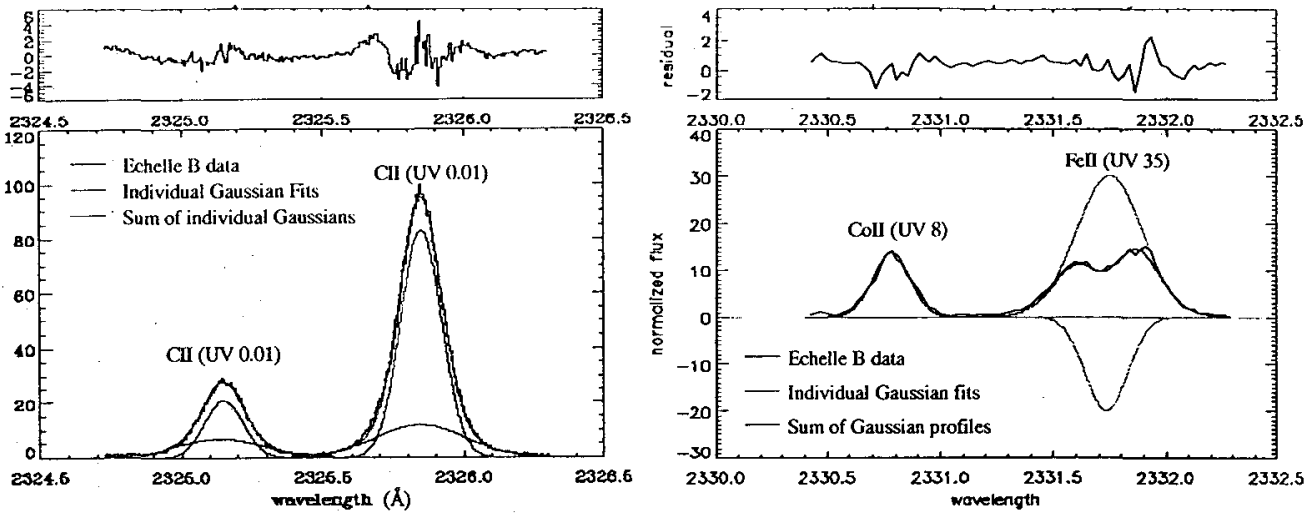

Figure 1. Gaussian fits to GHRS echelle line profiles of C II, Co II, and Fe II in the UV spectrum of $\alpha$ Tau. 

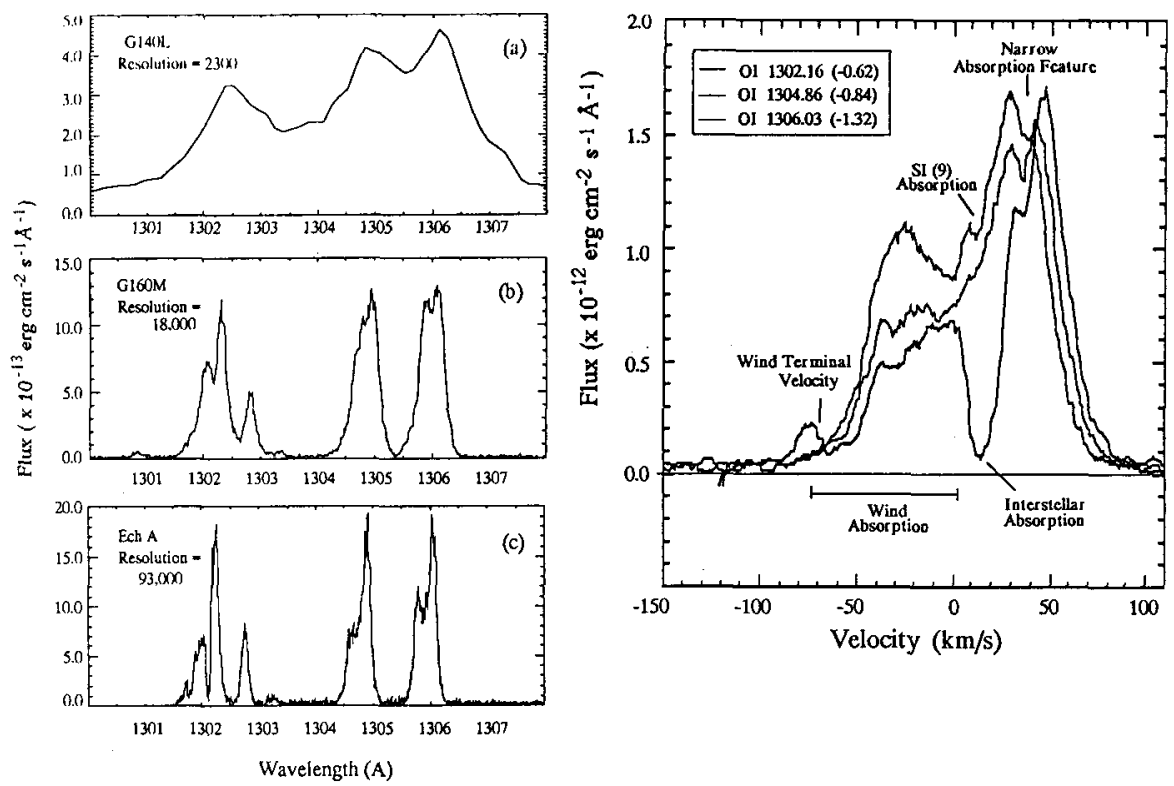

Figure 2. GHRS observations of the O I (UV 2) lines in the K-giant $\gamma$ Dra. Left: A comparison of low, medium, and echelle resolution spectra. Right: Echelle line profiles of the $\mathrm{O} I$ lines overplotted in velocity space.
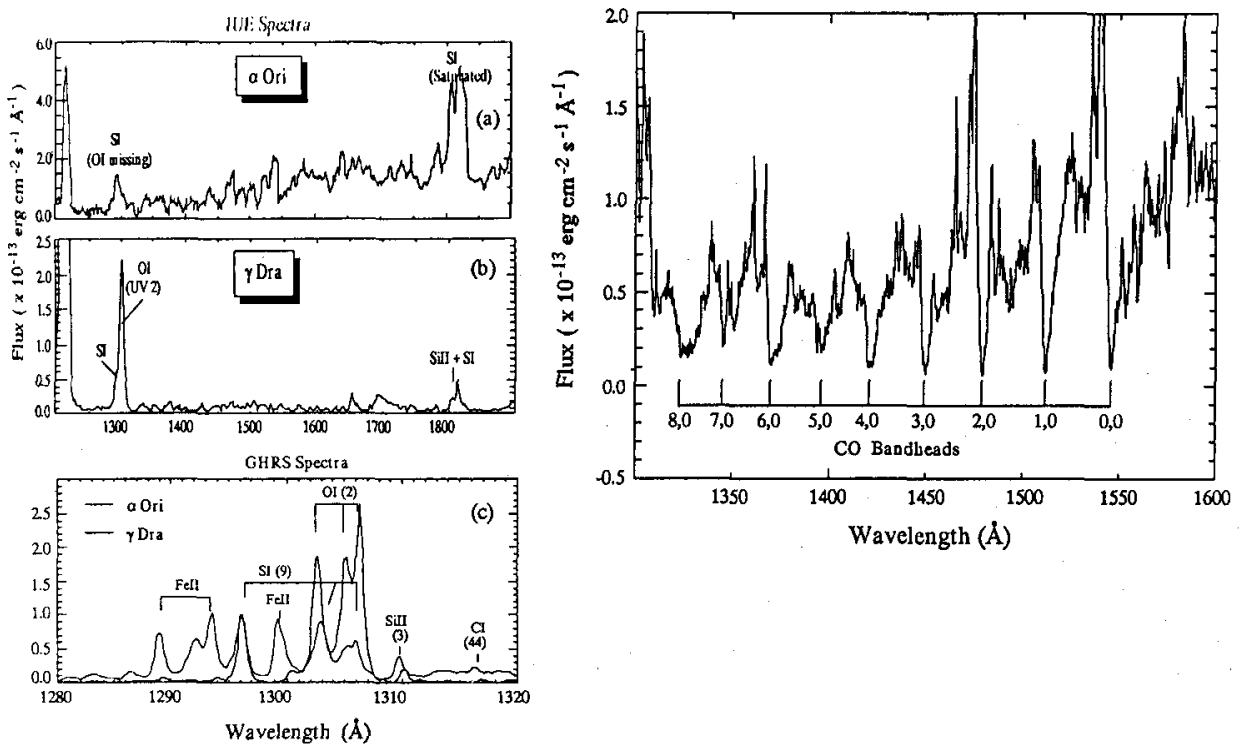

Figure 3. Left: IUE and GHRS low dispersion observations of $\alpha$ Ori and $\gamma$ Dra, illustrating the O I (UV 2) flux deficiency of the former. Right: GHRS G140L spectra of $\alpha$ Ori, showing the strong far-UV continuum and CO absorption bands. 